\title{
LA NIXTAMALIZACIÓN Y SU EFECTO EN EL CONTENIDO DE ANTOCIANINAS DE MAÍCES PIGMENTADOS, UNA REVISIÓN
}

\author{
THE NIXTAMALIZATION PROCESS AND ITS EFFECT ON ANTHOCYANIN \\ CONTENT OF PIGMENTED MAIZE, A REVIEW
}

\author{
Anayansi Escalante-Aburto ${ }^{1}$, Benjamín Ramírez-Wong ${ }^{1 *}$, Patricia I. Torres-Chávez ${ }^{1}$, \\ J. Manuel Barrón-Hoyos ${ }^{1}$, Juan de Dios Figueroa-Cárdenas ${ }^{2}$ y Jaime López-Cervantes ${ }^{3}$
}

\begin{abstract}
${ }^{1}$ Departamento de Investigación y Posgrado en Alimentos, Universidad de Sonora. Blvd. Luis Encinas y Rosales s/n. Apdo. Postal 1658. 83000, Hermosillo, Sonora. Tel y Fax 01 (662) 259-2207. ${ }^{2}$ Centro de Investigación y Estudios Avanzados (CINVESTAV- Unidad Querétaro). Libramiento Norponiente 2000, Fracc. Real de Juriquilla. 76230, Querétaro, Qro. ${ }^{3}$ Centro de Investigación e Innovación en Biotecnología Agropecuaria, Instituto Tecnológico de Sonora.Calle 6 de Abril esquina Chihuahua, Unidad Centro. 85130, Ciudad Obregón, Sonora.
\end{abstract}

${ }^{\star}$ Autor para correspondencia (bramirez@guaymas.uson.mx)

\section{RESUMEN}

El objetivo de esta revisión es compilar el conocimiento disponible acerca del contenido y los tipos de antocianinas identificadas en diferentes variedades de maíces (Zea mays L.) pigmentados. Principalmente, se pretende identificar el efecto en estos compuestos causado por la nixtamalización tradicional, y especificar los estudios desarrollados con tecnologías alternativas de nixtamalización para disminuir los efectos negativos en la calidad funcional del producto final. El uso de maíces pigmentados se ha incrementado debido al contenido superior de compuestos fenólicos, sobre todo por su contenido de antocianinas. Las antocianinas son los compuestos responsables del color rojo-azul de plantas como el maíz, y poseen propiedades antioxidantes benéficas para la salud. Estos pigmentos se encuentran en la capa de aleurona del endospermo y en el pericarpio del grano. Las antocianinas no aciladas de mayor abundancia en los maíces pigmentados son cianidina 3-glucósido (70 \%), pelargonidina 3-glucósido y peonidina 3-glucósido, las cuales poseen una alta capacidad antioxidante. En general, los maíces de variedades de color morado, azul y negro son las que contienen más antocianinas. La nixtamalización tradicional es un proceso agresivo para las antocianinas contenidas en los granos pigmentados, ya que provoca pérdidas hasta de $100 \%$ en los productos obtenidos por esta tecnología. No obstante, se puede retener una mayor cantidad de compuestos fenólicos, antocianinas y mayor capacidad antioxidante en productos obtenidos con nixtamalización por extrusión y nixtamalización fraccionada, los cuales son procesos alternativos eficientes a la nixtamalización tradicional. Sin embargo, son necesarias más investigaciones que permitan disminuir la pérdida de estos compuestos naturales útiles para la obtención de nuevos productos nutracéuticos desarrollados con maíces pigmentados.

Palabras clave: Zea mays, grano pigmentado, antocianinas, compuestos fenólicos, capacidad antioxidante, nixtamalización, extrusión.

\section{SUMMARY}

The purpose of this review is to compile available knowledge on content and anthocyanin type in different pigmented maize (Zea mays $\mathrm{L}$.) varieties. Additionally, the effects of traditional nixtamalization processing on these compounds are listed, and the studies performed to reduce the negative effects of traditional nixtamalization are described. The use of pigmented maize varieties has increased due to their high phenolic compound content, mainly anthocyanins. These compounds are responsible for the blue-red color in plants like maize, and they have antioxidant capacity. Anthocyanins are contained in the aleurone layer at the endosperm and in the pericarp of corn kernels. The non-acyl type anthocyanins, such as cyanidin 3-glucoside, pelargonidin 3-glucoside and peonidin 3 -glucoside are the most abundant compounds in colored maize; they have high antioxidant capacity. Generally, maize varieties with blue, purple and black color kernels contain the highest anthocyanin contents. Traditional nixtamalization processing is highly aggressive for anthocyanins in maize; it causes anthocyanin losses of up to $100 \%$ during traditional nixtamalization. However, alternative processes of nixtamalization, such as lime-cooking extrusion and fractionated nixtamalization can have higher anthocyanin and phenolic content retention. These newer nixtamalization processes can be more efficient than traditional nixtamalization. Nonetheless, more research is needed to further reduce losses of these natural compounds, which are useful for obtaining new nutraceutical products from pigmented maize varieties.

Index words: Zea mays, pigmented kernels, anthocyanins, phenolic compounds, antioxidant capacity, nixtamalization, extrusion.

\section{INTRODUCCIÓN}

Maíz (Zea mays L.), trigo (Triticum aestivum L.) y arroz (Oryza sativa L.) son los cereales más importantes del mundo, entre los cuales el maíz constituye la base de la alimentación para la mayor parte de los países en desarrollo. El maíz pertenece a la especie Zea de la familia de las gramíneas, y es una planta anual dotada de un amplio sistema radicular fibroso (FAO, 1993). La producción mundial anual de este cereal es de 800 millones de toneladas, y los principales productores son Estados Unidos, China, Brasil, Argentina, India, Francia e Indonesia (Singh et al., 2011). En México la producción de maíz en el año 2012 fue de 22069 $254 \mathrm{t}$ (SIAP, 2013). Sin embargo, actualmente no se cuenta con estadísticas oficiales acerca de la producción nacional y mundial de los maíces de color; solamente a nivel estatal existen datos reportados en Chiapas (Salinas et al., 2012), Estado de México (Salinas et al., 2010), Sinaloa y Tlaxcala (Agama-Acevedo et al., 2011). 
Las variedades criollas de maíz presentan granos pigmentados de múltiples colores, y se encuentran dentro de las 59 razas descritas (Sánchez et al., 2000). En los últimos años se ha incrementado el desarrollo y cultivo de otras variedades e híbridos pigmentados, como en Bolivia, Alemania, China, Estados Unidos y algunos países europeos. Debido al creciente interés por estos tipos de maíz en varias regiones del mundo, diversas instituciones gubernamentales y académicas investigan la mejora de las variedades existentes, incluyendo las de maíces criollos (Antonio et al., 2004).

Los colores de granos encontrados principalmente en las variedades pigmentadas son: amarillo, negro, morado, azul, rojo y naranja, aunque existen otras tonalidades. A estos maíces se les ha otorgado un valor agregado debido a su alto contenido de compuestos fenólicos del grupo de los flavonoides, entre los que destacan las antocianinas y los ácidos fenólicos (Masuoka et al., 2012). Además, en varios estudios se ha destacado el perfil de compuestos fenólicos, antocianinas y capacidad antioxidante de distintas variedades (Pedreschi y Cisneros-Zevallos, 2007; Li et al., 2008; Lee et al., 2010).

También se han adjudicado propiedades nutracéuticas a los maíces pigmentados por su alto contenido de antocianinas, las cuales poseen actividad biológica benéfica (antioxidante) derivada de sus metabolitos secundarios (Ruíz et al., 2008; Mora-Rochin et al., 2010; Mendoza-Díaz et al., 2012). Estos compuestos tienen una acción positiva en la salud al atacar radicales libres, los cuales se involucran en el desarrollo de enfermedades crónico-degenerativas (Liu, 2003; Gallardo et al., 2006; Arroyo et al., 2007).

El mercado de los alimentos nutracéuticos y alimentos funcionales se ha incrementado como resultado de la demanda de estos productos por parte de los consumidores (Lahance, 2002). En los países de América, los productos de maíz son los de mayor consumo, y la nixtamalización es el proceso principal al que se somete gran parte de la producción de esta gramínea. Por lo anterior, es importante hacer una búsqueda actualizada acerca de información sobre investigaciones realizadas en productos elaborados con maíces pigmentados y el proceso de nixtamalización.

El objetivo de este trabajo es revisar información relevante sobre los compuestos fenólicos, especialmente del contenido de antocianinas, en variedades de maíces pigmentados, así como del efecto de la nixtamalización tradicional (NT) sobre estos compuestos en los productos elaborados, y también del desarrollo de procesos de nixtamalización alternativos que promuevan una mayor retención de estos compuestos en el producto final.

\section{COMPUESTOS FENÓLICOS EN MAÍCES PIGMENTADOS}

Los compuestos fenólicos (CF) se incluyen dentro de un grupo de moléculas bioactivas no nutritivas (fitoquímicos) contenidas en frutas, vegetales y granos (Liu, 2004). El maíz contiene mayor cantidad de fenoles y capacidad antioxidante que otros cereales como trigo, arroz y avena (Ichikawa et al., 2001; Adom y Liu, 2002). Químicamente, un CF es una molécula que incluye en su estructura un anillo bencénico con uno o más grupos hidroxilo. Estos compuestos son derivados de sales 2-fenil-benzopirilo que existen en las plantas generalmente como glucósidos, con excepción de unos pocos compuestos amino.

Los CF comprenden una amplia variedad de formas químicas: fenoles simples, fenil-propanoides, derivados de ácido benzoico, flavonoides, estilbenos, taninos, lignanos y ligninas (Sahidi y Naczk, 2004). Los ácidos fenólicos se encuentran en los cereales en dos formas, libres y ligados (Dykes y Rooney, 2007). Las formas libres son menos abundantes en comparación con sus ésteres, glucósidos y compuestos ligados. En granos de cereales, su parte externa (pericarpio y testa) y la capa de células de aleurona en el endospermo contienen los fenoles libres en su forma glucosilada o esterificada (Yu et al., 2001). El contenido de ácidos fenólicos en el grano de maíz integral es de $601 \mathrm{mg}$ $\mathrm{kg}^{-1}$ (en base seca, bs), y el ácido ferúlico es el compuesto que constituye aproximadamente $63 \%$ de los fenoles totales (Mattila et al., 2004). Este compuesto se encuentra en el pericarpio en forma libre o esterificada a heteroxilanas, las cuales conforman la hemicelulosa de la pared celular del grano de maíz (Saulnier y Thibault, 1999).

Cabrera-Soto et al. (2009) demostraron que los CF del maíz se encuentran en varias partes anatómicas del grano donde varían en contenido, y que éste correlaciona con la dureza del grano. Estos autores también reportaron que el contenido de fenoles solubles e insolubles difirió entre dos híbridos de maíz blanco, y que en el germen de ambos hubo el mayor contenido de fenoles solubles.

Uno de los procesos para concentrar los CF provenientes de cereales, es mediante la decorticación de los granos para producir salvado, el cual puede ser incorporado como ingrediente en la elaboración de alimentos con mayor aporte de fibra dietaria y con propiedades nutracéuticas.

\section{Contenido de antocianinas en maíz}

Las antocianinas pertenecen a los flavonoides, un subgrupo importante de los CF, y son pigmentos ampliamente distribuidos en las plantas. En maíz, la conversión enzimática de 
las leucoantocianidinas a antocianidinas está controlada por el gen anthocyaninless2 (a2). La transformación de a2 en la capa de aleurona mediante bombardeo de partículas con el $\mathrm{ADN}_{\mathrm{c}}$ de $a 2$, conlleva a la producción de células pigmentadas (Cone, 2007).

El término antocianina es derivado de los vocablos griegos anthos (flor) y kyaneos (azul) (Liu, 2004; De PascualTeresa y Sánchez-Ballesta, 2008). Estos compuestos son los responsables de los colores azul, púrpura y rojo en plantas comestibles, principalmente cereales y frutas (EscribanoBailón et al., 2004). La forma aglicona de las antocianinas o antocianidina, posee una estructura basada en el ion flavilio o 2 -fenilbenzopirilium, que presenta grupos hidroxilo y metoxilo en diferentes posiciones (Figura 1). Consta de un esqueleto carbonado en forma C-6 (anillo A)- C-3 (anillo B)- C-6 (anillo C), cuya estructura forma un anillo cromano unido a un segundo anillo aromático (B) en la posición dos (Horbowicz et al., 2008). El tipo de antocianidina formado depende del grado o nivel de hidroxilación o metoxilación del anillo (B) de la molécula (Garzón, 2008).

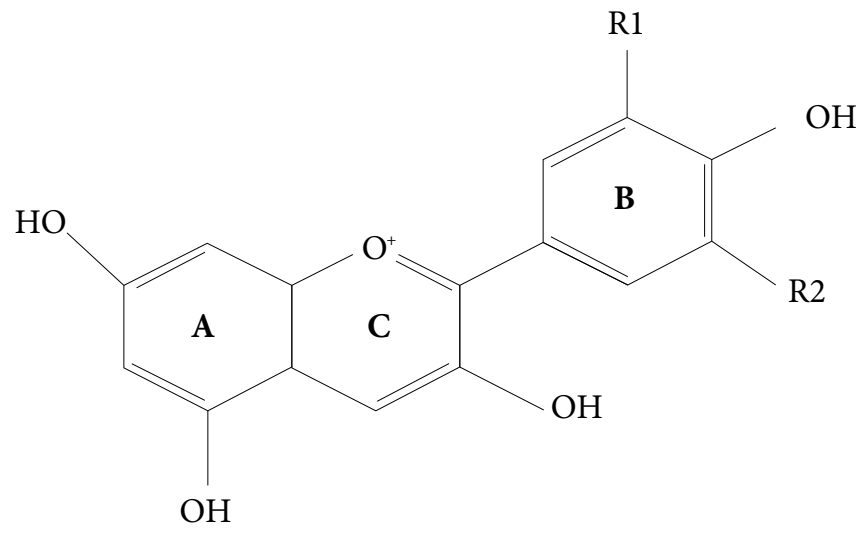

\begin{tabular}{lcc}
\hline Tipo de Antocianidina & $\mathrm{R} 1$ & $\mathrm{R} 2$ \\
\hline Cianidina & $\mathrm{OH}$ & $\mathrm{H}$ \\
Delfinina & $\mathrm{OH}$ & $\mathrm{OH}$ \\
Malvidina & $\mathrm{OCH}_{3}$ & $\mathrm{OCH}_{3}$ \\
Peonidina & $\mathrm{OCH}_{3}$ & $\mathrm{H}$ \\
Petunidina & $\mathrm{OH}$ & $\mathrm{OCH}_{3}$ \\
\hline
\end{tabular}

Figura 1. Estructura de antocianidinas en frutas y vegetales (Adaptado de De Pascual-Teresa y Sánchez-Ballesta, 2008).

El color de las antocianinas depende del número y posición de los grupos sustituyentes en la molécula. Un aumento en la cantidad de grupos hidroxilo produce pigmentos de tonos azules, y por el contrario, con predominancia de grupos metoxilo se presenta una tonalidad roja (Stintzing et al., 2002). Se han descrito de 12 a 17 antocianidinas diferentes, pero solo seis de ellas se encuentran comúnmente en frutas, vegetales y cereales como el maíz (Kong et al., 2003; De Pascual-Teresa y Sánchez-Ballesta, 2008).

Las antocianinas pueden encontrarse en forma acilada debido a la acilación de los residuos de azúcares con derivados de ácidos orgánicos alifáticos. Las formas no aciladas son menos estables que las antocianinas que presentan uno o más grupos acilo (De Pascual-Teresa et al., 2002).

En el Cuadro 1 se presentan los contenidos de antocianinas en diferentes variedades y tipos de maíz, clasificados en tres niveles: bajo, medio y alto. Como las antocianinas proporcionan el color característico a cada grano, los maíces blancos no aportan una cantidad significativa de estos compuestos, con contenidos de antocianinas entre $0.9 \mathrm{y}$ $1.59 \mathrm{mg} / 100 \mathrm{~g}$, en equivalentes de cianidina 3-glucósido (López-Martínez y García-Galindo, 2009; Hu y Xu, 2011). El contenido de antocianinas varía ampliamente entre genotipos; así, los maíces de color rojo presentan valores altos, medios y bajos, lo cual pudiera depender de su variabilidad genética. En general, los maíces que contienen bajos valores de antocianinas son los maíces amarillos y rosas, mientras que en el grupos de los valores medios se encuentran los maíces azules, y los contenidos más elevados ocurren en los granos de colores morado y negro.

Según López-Martínez y García-Galindo (2009) y López-Martínez et al. (2008), las variedades 'Veracruz 42' y 'AREQTL', ambas de color morado, presentan un elevado contenido de antocianinas en comparación con las demás variedades estudiadas. Además de conocer el tipo de antocianinas que contienen y su comportamiento durante diferentes tipos de procesamiento, resulta importante realizar una caracterización más completa y específica de los diferentes granos pigmentados.

\section{Tipos de antocianinas en maíces pigmentados}

Los primeros estudios enfocados a la caracterización de los pigmentos en maíz se realizaron hace más de 50 años. En flores y hojas de maíz se ha encontrado la presencia de cianidina 3-glucósido, cianidina 3-(6"-malonilglucósido), cianidina 3-(3", 6"dimalonilglucósido), peonidina 3-glucósido y peonidina 3-(dimalonilglucósido) (Harborne y Self, 1987; Fossen et al., 2001). Escribano-Bailón et al. (2004), en el "olote" (raquis de la inflorescencia femenina) del maíz morado, identificaron un compuesto correspondiente al dímero formado por condensación entre un compuesto del grupo flavan-3-ol y la cianidina 3,5-diglucósido.

En el grano de maíz se han encontrado pigmentos en pericarpio y en la capa de aleurona. En el endospermo del grano de maíz azul, la capa de aleurona contiene los pigmentos de antocianina azul que le confieren su color característico. 
Cuadro 1. Contenido de antocianinas en variedades de maíz con granos pigmentados.

Variedad de maíz Contenido de antocianinas $\pm \mathrm{DE}^{\dagger}$

(mg eq de cianidina 3 glucósido/100g)

\section{BAJO}

Amarillo ceroso (Xinnuo 301)

$0.63 \pm 0.05$

Amarillo comercial (Xinhuangdan 85)

Rojo carmesí

Rojo rubí

$1.11 \pm 0.03$

$5.09 \pm 0.17$

$6.94 \pm 0.19$

Negro

Rosa Jinheiyu

$10.07 \pm 1.766$

Fiesta India (Multicolor)

Rosa Cutie

Azul Cutie

Naranja

Azul suave comercial

$12.74 \pm 0.41$

$13.17 \pm 0.56$

$16.39 \pm 0.47$

$19.67 \pm 0.21$

$21.5 \pm 1.1$

$27.20 \pm 0.08$

Azul

Negro aperlado Guangdong

Azul americano comercial

Azul mexicano comercial

$29.15 \pm 1.44$

$29.22 \pm 0.86$

30.7

32.1

Azul Shaman

Amarillo "Población tropical"

Rojo púrpura dulce

Azul criollo

Azul comercial

Rojo comercial

RO (Rojo)

Morado

Mr-m (Rojo-morado)

Rojo carmesí Shijazhuang

M5IG04 (Rojo)

$32.27 \pm 0.15$

33.1

MEDIO

$60.71 \pm 2.17$

$62.09 \pm 0.84$

$63.1 \pm 1.4$

$82.3 \pm 3.8$

$127 \pm 2.13$

$127.7 \pm 0.49$

$141 \pm 1.56$

$149.3 \pm 5.63$

$159 \pm 1.22$

ALTO

Negro mexicano

$324 \pm 3.45$

Rojo mexicano

RaO04PV (Rojo)

$366 \pm 3.22$

$385 \pm 4.22$

NO04c (Negro)

$431 \pm 34.5$

Pinto mexicano (Rojo)

$431 \pm 3.89$

337 (Morado)

Nn04cl (Negro)

'Veracruz 42' mexicano (Morado)

'AREQTL' (Morado)

$478 \pm 4.11$

$529 \pm 7.12$

$1050 \pm 10.11$

$2050 \pm 10.15$

Morado oscuro Jingheinuo

$2565 \pm 112.1$

$3045 \pm 163.2$
Referencia

$\mathrm{Hu}$ y Xu (2011)

$\mathrm{Hu}$ y Xu (2011)

Abdel-Aal et al. (2006)

Abdel-Aal et al. (2006)

Agama-Acevedo et al. (2004)

Zhao et al. (2008)

Abdel-Aal et al. (2006)

Abdel-Aal et al. (2006)

Abdel-Aal et al. (2006)

López-Martínez y García-Galindo (2009)

Cortés-Gómez et al. (2006)

Agama-Acevedo et al. (2004)

Zhao et al. (2008)

Del Pozo-Insfran et al. (2006)

Del Pozo-Insfran et al. (2006)

Abdel-Aal et al. (2006)

Ruíz et al. (2008)

Abdel-Aal et al. (2006)

Escalante-Aburto et al. (2013)

López-Martínez et al. (2011)

López-Martínez et al. (2011)

López-Martínez y García-Galindo (2009)

Abdel-Aal et al. (2006)

López-Martínez y García-Galindo (2009)

Zhao et al. (2008)

López-Martínez y García-Galindo (2009)

López-Martínez y García-Galindo (2009)

López-Martínez y García-Galindo (2009)

López-Martínez y García-Galindo (2009)

López-Martínez y García-Galindo (2009)

López-Martínez y García-Galindo (2009)

López-Martínez y García-Galindo (2009)

López-Martínez y García-Galindo (2009)

López-Martínez y García-Galindo (2009)

López-Martínez y García-Galindo (2009)

Zhao et al. (2008)

Zhao et al. (2008)

${ }^{\dagger} \mathrm{DE}=$ desviación estándar. 
Estos compuestos derivan principalmente de la cianidina y pelargonidina, la primera identificada en maíces azules provenientes de Bolivia. También se ha sugerido la presencia de antocianinas en el germen de ciertas variedades de maíz (Betrán et al., 2001).

Nakatami et al. (1979) aislaron e identificaron las antocianinas de granos de maíz morado peruano, y encontraron como la principal antocianina a la cianidina 3-glucósido. Este compuesto se encuentra con mayor abundancia en el maíz, y se han descrito otras formas glucosiladas de esta antocianina, como pelargonidina, peonidina y delfinina (Mazza y Miniati, 1993). La cianidina 3-glucósido ha mostrado una alta capacidad antioxidante, incluso el doble que Trolox, un compuesto altamente antioxidante (Kuskoski et al., 2003).

Los maíces de color morado provenientes de Perú y Bolivia han sido los más estudiados. Al parecer, este tipo de coloración produce un mayor rendimiento de antocianinas al momento de su extracción, y una mayor capacidad antioxidante de las antocianinas contenidas en estos granos. Se han identificado tres tipos antocianinas no aciladas: la cianidina 3-glucósido, pelargonidina 3-glucósido y peonidina 3-glucósido (Yang et al., 2009). En este color de grano se ha identificado también a cianidina 3-(6"malonilglucósido), pelargonidina 3-(6"-malonilglucósido), peonidina 3-(6"-malonilglucósido), cianidina 3-(6"-etilmalonilglucósido), pelargonidina 3-(6"-etilmalonilglucósido) y peonidina 3-(6"-etilmalonilglucósido) (De Pascual-Teresa et al., 2002).

Por su parte, Aoki et al. (2002) reportaron, al igual que otros autores, a la cianidina 3-O- $\beta$-D-glucósido como la principal antocianina en extractos etanólicos obtenidos de maíz morado peruano. También confirmaron la presencia de otras cinco antocianinas más: pelargonidina 3-O- $\beta$ D-glucósido, peonidina 3-O- $\beta$-D-glucósido, cianidina 3-O- $\beta$-D-(6-malonil-glucósido), pelargonidina $3-O-\beta-\mathrm{D}-$ (6-malonil-glucósido) y peonidina $3-O-\beta$-D-(6-malonilglucósido). Estos autores concluyeron que los derivados de la cianidina constituyen aproximadamente el $70 \%$ de las antocianinas del grano. En diferentes provincias de China se ha detectado la presencia de cianidina 3-O-glucósido2-malonilglucósido, peonidina 3-(malonilglucósido) y la peonidina 3-(dimalonilglucósido), en cinco híbridos de maíces morados, y se comprobó que estos compuestos son estables en un rango amplio de tiempo y temperaturas, por lo que pudieran presentan mayor estabilidad durante su procesamiento (Zhao et al., 2008).

Es importante mencionar que la mayor parte de las in- vestigaciones se han enfocado al contenido de pigmentos en grano. Sin embargo, no se han considerado los contenidos de antocianinas y de compuestos fenólicos en el "olote", que también forma parte de los componentes de la planta y por lo general se considera como material de desecho. El maíz morado peruano posee mayor capacidad antioxidante y mejores cinéticas anti-radicales libres que los arándanos (Vaccinium myrtillus L.), con contenidos similares de antocianinas y compuestos fenólicos. En este mismo color del grano, el "olote" y el pericarpio también presentan una alta concentración de antocianinas, en comparación de ciertos tubérculos pigmentados (Cevallos-Casals y Cisneros-Zevallos, 2003). También se ha evidenciado la presencia de cianidina 3-glucósido, pelargonidina 3-glucósido, peonidina 3-glucósido y sus respectivos homólogos de malonil, en "olotes" de maíces morados cultivados en China (Yang y Zhai, 2010).

\section{NIXTAMALIZACIÓN DEL MAÍZ}

Este es el principal proceso de transformación del maíz para su consumo y fue desarrollado por los aztecas antes de la época precolombina: nixtamalización (del náhuatl, nextli, cal de cenizas; y tamalli, masa cocida de maíz) (Cabrera, 1992). Esta tecnología es aún utilizada ampliamente en América para la obtención de una gran variedad de productos (como tortillas, botanas, atoles, entre otros). El proceso de nixtamalización tradicional involucra cambios químicos, estructurales y nutricionales en los diversos constituyentes del grano (Gómez et al., 1989; Bressani, 1990; Serna-Saldívar et al., 1990; Ramírez-Wong et al., 1994; Rojas-Molina et al., 2007).

Actualmente se dispone de procesos alternativos a la nixtamalización tradicional (NT), como la nixtamalización por extrusión (NE) y la nixtamalización fraccionada (NF), que al igual que en la NT, los granos de maíz son sometidos a un tratamiento térmico-alcalino en presencia de exceso o limitada cantidad de agua.

El desarrollo de estos procesos alternativos se debe a que la NT requiere de largos periodos de tiempo y el uso de gran cantidad de agua, que da como subproducto al líquido denominado "nejayote" cuyo $\mathrm{pH}$ es elevado y contiene gran cantidad de material orgánico, al cual se le han encontrado pocos usos (González et al., 2004). Los procesos alternos se enfocan a la reducción de tiempo de procesamiento y de efluentes contaminantes; así, se han desarrollado tecnologías que optimizan el contenido de antocianinas en productos nixtamalizados para obtener un beneficio adicional a la salud de la población. 


\section{CAMBIOS EN EL CONTENIDO DE ANTOCIANINAS DE PRODUCTOS NIXTAMALIZADOS}

La estabilidad de las antocianinas depende principalmente de la presencia de luz, oxígeno, $\mathrm{pH}$, presencia de iones metálicos $\left(\mathrm{Ca}^{2+}\right)$ y de la temperatura (Bordignon-Luiz et al., 2007), y por ello los maíces que contienen estas biomoléculas son difíciles de procesar. Durante la NT y otros procesos alternos, ocurren varios de los factores antes mencionados. Los estudios que han evaluado los cambios en el contenido o perfil de antocianinas en productos elaborados mediante NT, se presentan a continuación. Posteriormente se discutirán los estudios realizados mediante otras tecnologías alternativas a la NT.

Durante el procesamiento por NT el maíz es sometido a condiciones de alto contenido humedad, calor $\left(80 \mathrm{a} 105^{\circ} \mathrm{C}\right)$ y un $\mathrm{pH}$ elevado (11 a 12). La NT reduce significativamente el contenido de antocianinas en los maíces pigmentados, pérdida que se debe a que gran cantidad de estos compuestos se solubilizan en el agua de cocción con $\mathrm{pH}$ elevado y temperatura extrema, lo que degrada a los compuestos. Además, otras estructuras químicas derivadas de los polifenoles son afectadas por el rompimiento de enlaces éster, y como consecuencia se liberan los fenoles a la solución de cocimiento. La mayor parte de estos compuestos se encuentran en el pericarpio del grano, y son eliminados durante el lavado del nixtamal (De la Parra et al., 2007).

Salinas-Moreno et al. (2003) evaluaron el efecto de la nixtamalización sobre las antocianinas en granos de variedades de maíz de colores rojo y azul producidos en ciertas regiones de México. Este estudio fue uno de los primeros que evaluó los cambios en el contenido y perfil de estos compuestos. Los autores reportaron que los maíces con pigmentos en el pericarpio presentaron mayores pérdidas (73 y $100 \%$ ), en contraste con los granos con pigmentos ubicados en la capa de aleurona que perdieron entre 19.5 y 50.2 $\%$, posiblemente por el efecto de protección del pericarpio a la capa de aleurona la cual mostró escaso daño. Además, los autores registraron un incremento en el contenido de cianidina 3-glucósido en harinas nixtamalizadas de maíz azul, y un cambio en el perfil de antocianinas de maíces de ambos colores, por una degradación de compuestos acilados que da lugar a la formación de nuevas estructuras químicas como antocianinas simples. Otros compuestos son prácticamente destruidos en su totalidad por efecto del $\mathrm{pH}$ alcalino.

Posteriormente, Salinas-Moreno et al. (2007) reportaron mayores contenidos de fenoles totales y libres en muestras de masas y tortillas nixtamalizadas mediante el método tradicional, comparados con los valores encontrados en el grano crudo, y también describieron algunos de los efectos de la NT en el oscurecimiento de masas y tortillas elaboradas con maíces comerciales.

Del Pozo-Insfran et al. (2006 y 2007) compararon la capacidad antioxidante y contenido de polifenoles (con énfasis en las antocianinas) de tortillas y "tortilla chips" elaboradas con maíz azul mexicano y un híbrido de maíz azul americano. Los contenidos de antocianinas para estos maíces fueron de 342 y $261 \mathrm{mg} \mathrm{kg}^{-1}$, respectivamente, y la pérdida de estos compuestos durante el procesamiento fue mayor en los productos derivados del maíz azul americano. Estos autores propusieron la adición de ácido fumárico ( $\mathrm{pH}$ 5.2) a las masas de maíz nixtamalizado, para mejorar la retención de antocianinas, fenoles solubles y capacidad antioxidante. Con esta técnica se logró retener una mayor cantidad de los compuestos de interés, además de que se presentó mayor capacidad antioxidante y una mejor coloración en los productos obtenidos.

Por su parte, López-Martínez et al. (2011) evaluaron el efecto de la NT sobre el contenido de antocianinas y la capacidad antioxidante en tortillas elaboradas con maíz blanco, azul, rojo y morado. Encontraron una disminución en el contenido de antocianinas y fenoles debida al tratamiento térmico alcalino. Sin embargo, la masa y tortilla del maíz morado 'Veracruz 42' presentaron una mayor capacidad antioxidante, lo que atribuyeron al elevado contenido de antocianinas y fenoles presentes en esta variedad de maíz, demostrando así que la pérdida de antocianinas está relacionada con la variedad de maíz utilizada. Estos autores también reportaron un incremento en la capacidad antioxidante al cocer la masa para obtención de tortillas, debido probablemente al aumento de fenoles solubles.

Agama-Acevedo et al. (2004) reportaron que el almidón de maíz azul contiene más antocianinas totales que el maíz negro, ya que en el maíz azul los pigmentos se encuentran distribuidos en pericarpio, capa de aleurona y endospermo. Pero el contenido de antocianinas en maíz negro nixtamalizado resultó mayor que el azul sometido al mismo proceso, debido a que el pericarpio permaneció casi intacto y porque posiblemente este maíz tiene mayor contenido de antocianinas aciladas que deriven de la petunidina, como petanina que es más estable a la nixtamalización. En adición, las antocianinas del endospermo en el maíz azul presentaron mayor exposición a la solución alcalina, ya que las proteínas que forman parte de la subaleurona resultaron solubilizadas durante el procesamiento.

Mendoza-Díaz et al. (2012) evaluaron contenido de antocianinas, capacidad antioxidante y antimutagénica en tortillas elaboradas mediante la NT, en granos de maíces pigmentados criollos de varios colores (blanco, amarillo, rojo y azul). Estos autores encontraron resultados similares a los 
descritos anteriormente respecto a la pérdida de antocianinas durante el procesamiento del grano a masa $(83 \%)$ y tortilla (64\%); sin embargo, después de la nixtamalización los maíces azules y rojos superaron a las demás variedades en contenido de antocianinas.

\section{PROCESOS ALTERNOS DE NIXTAMALIZACIÓN}

A través de los años diversos investigadores han desarrollado nuevos procesos de nixtamalización (Martínez-Bustos et al., 1996; Martínez-Flores et al., 1998; Cortés-Gómez et al., 2005; Figueroa et al., 2006; Cuevas-Rodríguez et al., 2009; Ortega et al., 2011).

La nixtamalización fraccionada (NF) fue desarrollada por Cortés-Gómez et al. (2006) para la elaboración de harinas nixtamalizadas. En esta técnica, los granos de maíz se remojan en agua a $30^{\circ} \mathrm{C}$ durante $5 \mathrm{~min}$, luego se introducen a un prototipo de decorticador con una velocidad de tornillo de $800 \mathrm{rpm}$. La separación del endospermo y de las otras partes del grano se basa en la diferencia de densidades del pericarpio, germen y pedicelo. Las fracciones separadas y el endospermo se nixtamalizan a $90{ }^{\circ} \mathrm{C}$ en soluciones de hidróxido de calcio. Finalmente, las fracciones se secan en un secador de charola y se muelen. Con la NF se demostró que la NT es un proceso más agresivo para las antocianinas de harinas de maíz azul (pérdida de $95 \%$, con respecto al contenido en grano) que la NF, debido probablemente a que la capa del endospermo y del embrión son removidas y nixtamalizadas por separado, lo que permite obtener harinas y tortillas con mayor cantidad de antocianinas (retención de $58 \%$ con respecto al grano).

El proceso de extrusión también se utiliza para la producción de harinas nixtamalizadas con las que se elaboran subproductos como masa, tortillas y botanas. La nixtamalización por extrusión (NE) se ha aplicado para la obtención de tortillas de maíces azul y rojo, para ser comparados con sus similares obtenidos mediante la NT. La NE pudo retener mayor cantidad de antocianinas en tortillas elaboradas con maíces de color amarillo y rojo, mientras que las tortillas de maíz azul perdieron la misma cantidad de antocianinas que las procesadas con la NT (Mora-Rochin et al., 2010). Aquí conviene destacar que el contenido de antocianinas en el maíz azul fue significativamente mayor que en los granos rojos, amarillos y blancos.

Aguayo-Rojas et al. (2012) evaluaron el contenido de antocianinas y capacidad antioxidante de tortillas obtenidas de harinas obtenidas por NE de maíces mexicanos de varios colores (blanco, amarillo, rojo y azul). Al igual que lo reportado por otros autores, el maíz azul presentó el contenido de antocianinas más elevado que los demás maíces estudiados (275.2 $\mathrm{mg} \mathrm{kg}^{-1}$, equivalentes de cianidina 3-glu- cósido, bs), con una pérdida de estos compuestos de $53.5 \%$ en las tortillas extrudidas. En esta investigación se concluyó que el cocimiento alcalino por extrusión permite una mayor retención de compuestos fenólicos y, por tanto, mayor capacidad antioxidante en las tortillas.

En la obtención de extrudidos nixtamalizados expandidos para producir botanas, se optimizan las variables del proceso de extrusión y del contenido de $\mathrm{Ca}(\mathrm{OH})_{2}$, para obtener botanas con mayor contenido de antocianinas, y con una textura similar a la de productos encontrados en el mercado.

Escalante-Aburto et al. (2013) evaluaron diferentes condiciones de procesamiento de la NE, haciendo variar la humedad de alimentación, temperatura y tamaño de partícula del maíz molido, para obtener extrudidos expandidos nixtamalizados de maíz azul tipo botana con buenas características físicas, de textura y con mayor cantidad de antocianinas. El maíz azul presentó un alto contenido de estos compuestos (620.9 mg kg-1 equivalentes de cianidina 3-glucósido, bs). Con todos los tratamientos evaluados se logró obtener una botana con características físicas y de textura aceptables, que retuvieron $38.5 \%$ de las antocianinas totales contenidas en el grano y con un incremento de $11.3 \%$ en el contenido de cianidina 3-glucósido, lo que concuerda con lo reportado por Salinas-Moreno et al. (2003). Estos autores concluyeron que es posible la optimización de este proceso para desarrollar una botana con características organolépticas aceptables y con alto contenido de antocianinas.

\section{CONCLUSIONES}

El interés por el estudio, desarrollo y producción de alimentos de maíces pigmentados se ha incrementado durante los últimos años. Gracias a tales estudios se ha corroborado que la cianidina 3-glucósido es la antocianina más abundante en estos maíces, seguida de la pelargonidina 3-glúcosido y peonidina 3-glucósido, las cuales corresponden al grupos de antocianinas no aciladas. Además, se ha detectado la presencia de un gran número de compuestos acilados en los granos de maíz.

Durante la nixtamalización del grano se pierde gran cantidad de estos compuestos, porque se degradan mediante solubilización, por la remoción del pericarpio y por la labilidad térmica, especialmente durante la nixtamalización tradicional. Las nuevas opciones de nixtamalización, la fraccionada y la de extrusión, han mostrado que es posible retener mayor cantidad de antocianinas (de 38 a $58 \%$ ) en los productos desarrollados con estas tecnologías. Adicionalmente, durante el procesamiento se forman diferentes compuestos o se incrementa el contenido de algunos ya 
existentes, lo que cambia el perfil de antocianinas.

En la actualidad se continúan mejorando y desarrollando nuevas tecnologías de procesamiento, con el fin de evitar la pérdida de antocianinas y la obtención de productos de maíz con valor nutrimental agregado, además de la introducción de nuevas formas de consumo de maíces pigmentados. Existe la necesidad de continuar con estudios que permitan el desarrollo de métodos de procesamiento para retener la mayoría de los compuestos fenólicos contenidos en los granos, con la finalidad de aprovechar su potencial benéfico en la salud de los consumidores de este tipo de productos.

\section{AGRADECIMIENTOS}

Al CONACYT por el financiamiento para estudios de doctorado de Escalante-Aburto, y a la Dra. María Lourdes Aldana Madrid y al M.C. Néstor Ponce García por el apoyo técnico brindado.

\section{BIBLIOGRAFÍA}

Abdel-Aal E S, C J Young, I Rabalski (2006) Anthocyanin composition in black, blue, pink, purple, and red cereal grains. J. Agric. Food Chem. 54:4696-4704.

Adom K K, R H Liu (2002) Antioxidant activity of grains. J. Agric. Food Chem. 50:6182-6187.

Agama-Acevedo E, M A Ottenhof, M I Farhat, O Paredes-López, J Ortíz-Cereceres, L A Bello-Pérez (2004) Efecto de la nixtamalización sobre las características moleculares del almidón de variedades pigmentadas de maíz. Interciencia 29:643-649.

Agama-Acevedo E, Y Salinas-Moreno, G Pacheco-Vargas, L A BelloPérez (2011) Características físicas y químicas de dos razas de maíz azul: Morfología del almidón. Rev. Mex. Cienc. Agríc. 2:317-329.

Aguayo-Rojas J, S Mora-Rochín, E O Cuevas-Rodríguez, S O SernaSaldívar, J A Gutiérrez-Uribe, C Reyes-Moreno, J Milán-Carrillo (2012) Phytochemicals and antioxidant capacity of tortillas obtained after lime-cooking extrusion process of whole pigmented mexican maize. Plant Food Hum. Nutr. 67:178-185.

Antonio M M, J L Arellano V, G García de los Santos, S Miranda Colín, J A Mejía Contreras, F V González Cossío (2004) Variedades criollas de maíz azul raza chalqueño. Características agronómicas y calidad de semilla. Rev. Fitotec. Mex. 27:9-15.

Aoki H, N Kuze, Y Kato (2002) Anthocyanin isolated from purple corn (Zea mays L.) Disponible en: http://www.ffcr.or.jp/zaidan/ffcrhome.nsf/7bd44c20b0dc562649256502001b65e9/c66987733 61b42b249256ba60018e581/\$FILE/anthocyanin-FFIJ199.pdf (Agosto 2013)

Arroyo J, E Raez, M Rodríguez, V Chumpitaz, J Burga, W De la Cruz, J Valencia (2007) Reducción del colesterol y aumento de la capacidad antioxidante por el consumo crónico de maíz morado (Zea mays L.) en ratas hipercolesterolémicas. Rev. Peruana Med. Exp. Salud Púb. 24:157-162.

Betrán F J, A J Bockholt, L W Rooney (2001) Blue corn. In: Specialty Corns. A R Hallauer (ed). Iowa State University, Ames Iowa, USA. pp:293-337.

Bordignon-Luiz M T, C Gauche, E F Gris, L D Falcao (2007) Colour stability of anthocyanins from Isabel grapes (Vitis labrusca L.) in model systems. LWT-Food Sci. Technol. 40:549-599.

Bressani R (1990) Chemistry, technology and nutritive value of maize tortillas. Food Rev. Int. 6:225-264.

Cabrera-Soto M L, Y Salinas-Moreno, G A Velázquez-Cardelas, E Espinosa-Trujillo (2009) Content of soluble and insoluble phe- nols in the structures of corn grain and their relationship with physical properties. Agrociencia 43:827-839.

Cabrera L (1992) Diccionario de Aztequismos. COLOFON, México, D. F. Cevallos-Casals B A, L Cisneros-Zevallos (2003) Stoichiometric and kinetic studies of phenolics antioxidants from Andean purple corn and red-fleshed sweetpotato. J. Agric. Food Chem. 51:3313-3319.

Cone K C (2007) Anthocyanin synthesis in maize aleurone tissue. Plant Cell Monogr. 8:121-139.

Cortés-Gómez A, E San Martín-Martínez, F Martínez-Bustos, G M Vázquez-Carrillo (2005) Tortillas of blue maize (Zea mays L.) prepared by a fractionated process of nixtamalization: Analysis using response surface methodology. J. Food Eng. 66:273-281.

Cortés-Gómez A, Y Salinas M, E San Martín-Martínez, F MartínezBustos (2006) Stability of anthocyanins of blue maize (Zea mays L.) after nixtamalization of separated pericarp-germ tip cap and endosperm fractions. J. Cereal Sci. 43:57-62.

Cuevas-Rodríguez E O, C Reyes-Moreno, S Eckhoff R, J Milán-Carrillo (2009) Nixtamalized instant flour from corn (Zea mays L.) meal: Optimization of nixtamalization conditions. Cereal Chem.86:7-11.

De la Parra C, S O Serna S, R Hai L (2007) Effect of processing on the phytochemical profiles and antioxidant activity of corn for production masa, tortillas, and tortilla chips. J. Agric. Food Chem. 55:4177-4183.

De Pascual-Teresa S, C Santos-Buelga, J C Rivas-Gonzalo (2002) LCMS analysis of anthocyanins from purple corn cob. J. Sci. Food Agric. 82:1003-1006.

De Pascual-Teresa S, M T Sánchez-Ballesta (2008) Anthocyanins: from plant to health. Phytochem. Rev. 7:281-299.

Del Pozo-Insfran D, C H Brenes, S O Serna S, S T Talcott (2006) Phenolic and antioxidant content of White and blue corn (Zea mays L.) products. Food Res. Int. 39:696-703.

Del Pozo-Insfran D, S O Serna S, C H Brenes, S T Talcott (2007) Polyphenolics and antioxidant capacity of white and blue corns processed into tortillas and chips. Cereal Chem. 84:162-168.

Dykes L, L W Rooney (2007) Phenolic compounds in cereal grains and their health benefits. Cereal Foods World 52:105-111.

Escalante-Aburto A, B Ramírez-Wong, P I Torres-Chávez, J D Figueroa Cárdenas, J López-Cervantes, J M Barrón-Hoyos, I MoralesRosas (2013) Effect of extrusion processing parameters on anthocyanin content, physicochemical properties of nixtamalized blue corn expanded extrudates. CyTA-J. Food 11:29-37.

Escribano-Bailón M T, C Santos-Buelga, J C Rivas-Gonzalo (2004) Anthocyanins in cereals. J. Chromatogr. 1054:129-141.

FAO, Food and Agriculture Organization (1993) El Maíz en la Nutrición Humana. Organización de las Naciones Unidas, Roma. http:// www.fao.org/docrep/t0395s/T0395S02.htm (Diciembre, 2013).

Figueroa C J D, A Rodríguez C, J J Véles M (2006) Proceso ecológico de nixtamalización para la producción de harinas masas y tortillas integrales. Mexican Patent PA/a/2005/011797.

Fossen T, R Slimestad, O M Andersen (2001) Anthocyanins from maize (Zea mays L.) and reed canarygrass (Phalaris arundinacea). J. Agric. Food Chem. 49:2318-2321.

Gallardo C, L Jiménez, M T García-Conesa (2006) Hydroxycinnamic acid composition and in vitro antioxidant activity of selected grain fractions. Food Chem. 99:455-463.

Garzón G A (2008) Anthocyanins as natural colorants and bioactive compounds. A review. Acta Biol. Colomb. 13:27-36.

Gómez M H , C M McDonough, L W Rooney, R D Wanishka (1989) Changes in corn and sorghum during nixtamalization and tortilla baking. J. Food Sci. 53:330-336.

González R, E Reguera, L Mendoza, J M Figueroa, F Sánchez-Sinecio (2004) Physicohemical changes in the hull of corn grains during their alkaline cooking. J. Agric. Food Chem. 52:3831-3837.

Harborne J B, R Self (1987) Malonated cianidin 3-glucosides in Zea mays and other grasses. Phytochemistry 26:2417-2418.

Horbowicz M, R Kosson, A Grzersiuk, H Debski (2008) Anthocyanins of fruits and vegetables, their occurrence, analysis a role in human nutrition. Vegetable Crops Res. Bull. 68:5-22.

Hu Q, Xu J (2011) Profiles of carotenoids, anthocyanins, phenolics, and antioxidant activity of selected color waxy corn grains during maturation. J. Agric. Food Chem. 59:2026-2033. 
Ichikawa H, T Ichiyanagi, B Xu, Y Yoshii, M Nakajima, T Konishi (2001) Antioxidant activity of anthocyanin extract from purple black rice. J. Med. Food 4:211-218.

Kong J M, L S Chia, N K Goh, T F Chia, R Brouillard (2003) Analysis and biological activities of anthocyanins. Phytochemistry 64:923-933.

Kuskoski E M, A G Asuero, M C García-Parilla, A M Troncoso, R Fett (2003) Actividad antioxidante de pigmentos antociánicos. Cien. Tecnol. Alim. Campinas 24:691-693.

Lahance P A (2002) Nutraceuticals, for real nutraceuticals and functional foods. Food Technol. 56:20.

Lee C H, H S García, K L Parkin (2010) Bioactivities of kernel extracts of 18 strains of maize (Zea mays L). J. Food Sci. 75:C667-C672.

Li C Y, H W Kim, S R Won, H K Min, K J Park, J Y Park, M S Ahn, H I Rhee (2008) Corn husk as potential source of anthocyanins. J. Agric. Food Chem. 56:11413-11416.

Liu R H (2003) Health benefits of fruits and vegetables from additive and synergistic combination of phytochemicals. Am. J. Clin. Nutr. 78:517S-520S.

Liu R H (2004) Potential synergy of phytochemicals in cancer prevention: Mechanism of action. Am. J. Clin. Nutr. 134:3479S-3485S.

Lopez-Martinez L X, R M Oliart-Ros, G Valerio-Alfaro, C H Lee, K L Parkin, H S García (2008) Antioxidant activity, phenolic compounds and anthocyanins content of eighteen strains of Mexican maize. LWT-Food Sci. Techol. 42:1187-1192.

López-Martínez L X, H S García-Galindo (2009) Actividad antioxidante de extractos metanólicos y acuosos de distintas variedades de maíz mexicano. Nova Scientia 3,2:51-65.

Lopez-Martinez L X, K L Parkin, H S García (2011) Phase II-Inducing, Polyphenols content and antioxidant capacity of corn (Zea mays L.) from phenotypes of white, blue, red, and purple colors processed into masa and tortillas. Plant Food Hum. Nutr. 66:41-47.

Mattila P, J M Pihlava, J Hellström (2004) Contents of phenolic acids, alkyl and alkenylresorcinols, and avenanthramides in commercial grain products. J. Agric. Food Chem. 53:8290-8295.

Martinez-Bustos F, J D Figueroa-C, J L Martinez, F S Sinencio, J Gonzalez-Hernandez, J L Montes M, M Ruiz-Torres (1996) Extrusion Apparatus for the Preparation of Instant Fresh Corn Dough or Masa. United States Patent Number 5558 886. Date of Patent Sep 24, 1996.

Martínez-Flores H E, F Martínez-Bustos, J D Figueroa C, J GonzálezHernández (1998) Tortillas from extruded masa as related to corn genotype and milling process. J. Food Sci. 63:130-133.

Masuoka N, M Matsuda, I Kubo (2012) Characterisation of the antioxidant activity of flavonoids. Food Chem. 131:541-545.

Mazza G, E Miniati (1993) Anthocyanins in Fruits, Vegetables, and Grains. CRC Press, London, UK. 362 p.

Mora-Rochin S, J A Gutiérrez-Uribe, S O Serna-Saldívar, P SánchezPeña, C Reyes-Moreno, J Milán-Carrillo (2010) Phenolic content and antioxidant activity of tortillas produced from pigmented maize processed by conventional nixtamalization cooking. J. Cereal Sci. 52:502-508.

Mendoza-Díaz S, M C Ortíz-Valerio, E Castaño-Tostado, J D FigueroaCárdenas, R Reynoso-Camacho, $M$ Ramos-Gómez, R Campos-Vega, G F Loarca-Piña (2012) Antioxidant capacity and antimutagenic activity of anthocyanin and carotenoid extracts from nixtamalized pigmented creole maize races (Zea mays L.). Plant Food Hum. Nutr. 67:442-449.

Nakata N, H Fukuda, H Fuwa (1979) Major anthocyanins of Bolivian purple corn (Zea mays L.). Agric. Biol. Chem. 43:389-391.

Ortega M J, E Morales-Sánchez, M A Hernández-Román, M Ruíz-Torres (2011) Sistema para producir harina nixtamalizada con transporte de bajo cizallamiento. Ing. Mec. Tecnol. Desarrollo
4:33-42.

Pedreschi R, L Cisneros-Zevallos (2007) Phenolic profiles of Andean purple corn (Zea mays L.). Food Chem. 100:965-963.

Ramírez-Wong B, V E Sweat, P I Torres, L W Rooney (1994) Cooking time, grinding, and moisture content effect on fresh corn masa texture. Cereal Chem. 71:337-343.

Rojas-Molina I, E Gutiérrez-Cortez, A Palacios-Fonseca, L Baños, J I Pons-Hernandez, S H Guzmán-Maldonado, P Pineda-Gomez, M E Rodríguez (2007) Study of structural and thermal changes in endosperm of quality protein maize during traditional nixtamalization process. Cereal Chem. 84:304-312.

Ruíz T N A, F Rincón S, V M Hernández L, J D Figueroa C, F M G Loarca P (2008) Determinación de compuestos fenólicos y su actividad antioxidante en granos de maíz. Rev. Fitotec. Mex. 31:29-34.

Sahidi F, M Naczk (2004) Phenolics in food and nutraceuticals, CRC Press LLC, Boca Raton, FL. 566 p.

Salinas-Moreno Y, F Martínez-Bustos, M Soto-Hernández, R OrtegaPaczka, J L Arellano-Vázquez (2003) Efecto de la nixtamalización sobre las antocianinas de granos pigmentados. Agrociencia 37:617-628.

Salinas-Moreno Y, J J López-Reynoso, G González-Flores, G VázquezCarrillo (2007) Compuestos fenólicos del grano de maíz y su relación con el oscurecimiento de masa y tortilla. Agrociencia 41:295-305

Salinas M Y, J Soria R, E Espinosa T (2010) Aprovechamiento y distribución de maíz azul en el Estado de México, $1^{\text {a }}$ ed. Instituto Nacional de Investigaciones Forestales, Agrícolas y Pecuarias, Folleto Técnico Núm. 42

Salinas M Y, F J Cruz C, S A Díaz, O, F Castillo G (2012) Granos de maíces pigmentados de Chiapas, características físicas, contenido de antocianinas y valor nutracéutico. Rev. Fitotec. Mex. 35:33-41.

Sánchez G J J, M M Goodman, C W Stuber (2000) Isozymatic and morphological diversity in the races of maize of Mexico. Econ. Bot. 54:43-59.

Saulnier L, J F Thibault (1999) Ferulic acid and diferulic acids as components of sugar-beet pectins and maize bran heteroxylans. J. Sci. Food Agric. 79:396-402.

Serna-Saldivar S O, D A Knabe, L W Rooney, T D Tanksley Jr (1990) Effect of lime cooking on energy and protein digestibility of maize and sorghum. Cereal Chem. 64:252-257.

SIAP, Servicio de Información Agroalimentaria y Pesquera(2013) http://www.siap.gob.mx/index.php?option $=$ com wrapper\&view $=$ wrapper\&Itemid=350 (Agosto 2013).

Singh N, S Singh, K Shevkani (2011) Maize: Composition, bioactive constituents, and unleavened bread. In: Flour and Breads and Their Fortification in Health and Disease Prevention. V J Preedy, W R Ross, P V Patel (eds). Elsevier Inc. California, USA. pp:89-95.

Stintzing F C, A S Stintzing, R Carle, B Frei, R E Wrolstad (2002) Color and antioxidant properties of cyanidin-based anthocyanin pigments. J. Agric. Food Chem.50:6172-6181.

Yang Z, W Zhai (2010) Identification and antioxidant activity of anthocyanins extracted from the seed and cob of purple corn (Zea mays L.). Innov. Food Sci Emerg. Technol.11:169-176.

Yang Z, Z Chen, S Yuan, W Zhai, X Piao, X Piao (2009) Extraction and identification of anthocyanins from purple corn (Zea mays L.) Int .J. Food Sci. Technol. 44:24-85-2492.

Yu J, T Vasanthan, F Temelli (2001) Analysis of phenolic acids in barley by high-performance-liquid-chromatography. J. Agric. Food Chem. 49:4352-4358.

Zhao X, M Corrales, C Zhang, X Hu, Y Ma, B Tauscher (2008) Composition and thermal stability of anthocyanins from Chinese purple corn (Zea mays L.). J. Agric. Food Chem. 56:10761-10766. 